\title{
The Zika Virus and Pregnancy
}

\author{
D. G. Fiorentino ${ }^{1}$ - F. J. Montero ${ }^{1}$
}

Published online: 13 July 2016

(C) Springer Science+Business Media New York 2016

\begin{abstract}
Purpose of Review Following the recent association of Zika virus with microcephaly in Brazil, there has been a multitude of studies attempting to elucidate the relationship between Zika virus infection in pregnancy and congenital anomalies. The American Congress of Obstetricians and Gynecologists (ACOG), the U.S. Centers for Disease Control and Prevention (CDC), and the Society for Maternal Fetal Medicine (SMFM) have all issued guidelines governing the screening and management of pregnant patients with Zika exposure. These guidelines have rapidly evolved as the scientific evidence supporting causation of microcephaly in cases of Zika Virus infection has continued to mount. The purpose of this article is to review the current guidelines and the available evidence on which they are based.

Recent Findings A series of experiments on animals and human cell lines have demonstrated that Zika virus is neurotropic. Further, examinations of amniotic fluid, fetal tissues, and placenta have confirmed the ability of Zika virus to cross the placenta. With the currently available evidence, the CDC has recently concluded that Zika virus infection in pregnancy is a cause of microcephaly.

Summary Recently, it has become clear that Zika virus infection during pregnancy is responsible for congenital brain anomalies and microcephaly in the offspring. What remains unclear is the rate of vertical transmission from mother to
\end{abstract}

This article is part of the Topical Collection on High-risk Gestation and Prenatal Medicine

F. J. Montero

freddyjmd@gmail.com

1 Department of Obstetrics and Gynecology, Miller School of Medicine, University of Miami, 1611 NW 14 Street, Holtz Children's Hospital \#4070, Miami, FL 33136, USA fetus, the risk of microcephaly, and other CNS anomalies in fetuses when maternal infection is proven and the time from exposure or infection to clinical manifestations including ultrasound anomalies. Further studies are needed to answer these important questions.

Keywords Zika virus $\cdot$ Microcephaly $\cdot$ Congenital brain anomalies $\cdot$ Pregnancy

\section{Introduction}

Zika virus is a member of the flavivirus family and, as such, is related to other well-known flaviviruses, including those that cause dengue, yellow fever, and West Nile infections. It is carried by the Aedes aegypti mosquito, which lives predominantly in temperate regions and can also transmit the dengue and chikungunya viruses. The virus was first discovered in the Zika forest of Uganda in the 1940s. Since its initial isolation, multiple outbreaks have been reported in Africa, Southeast Asia, the Pacific Islands, and most recently the Americas.

Infection with Zika virus is asymptomatic in approximately $80 \%$ of cases [1]. When symptoms do occur in healthy, nonpregnant patients, they are typically mild and self limiting and include rash, arthralgia, conjunctivitis, and fever. Typically, symptoms resolve with only supportive care within 2 to 7 days. The majority of cases go undetected as less than $20 \%$ of individuals infected with Zika are symptomatic and hospitalization and mortality rates related to infection are low [2•]. In pregnant women, however, Zika infection has recently been associated with birth defects prompting the World Health Organization (WHO) to declare a Public Health Emergency of International Concern [3]. In 2015, the Ministry of Health in Brazil reported a 20 -fold increase in the incidence of 
microcephaly, particularly in the regions most affected by Zika virus infections.

\section{Transmission and Prevention}

Current evidence suggests that the Zika virus can be transmitted not only through the bite of an infected mosquito but also via sexual contact with an infected male partner, blood transfusion, or vertical maternal-fetal transmission prenatally or intrapartum. Thus far, the most common mechanism of transmission is mosquito borne.

\section{Vector-Borne Transmission}

During the first weeks of Zika infection, the virus can be isolated from the bloodstream. When a mosquito bites an infected person during this period of viremia, it acquires the virus and can then spread it to others. The Aedes mosquitoes that carry Zika feed primarily on humans, often bite multiple humans in a single meal, and live in close proximity to human habitation [4]. They are known to bite primarily during the day, typically lay eggs in and near standing water, and can be found throughout much of the Americas.

\section{Prevention of Vector-Borne Transmission}

The most important method of prevention is avoidance of exposure. When possible, pregnant women should delay travel to areas with active Zika transmission [5••]. When travel is unavoidable, women are encouraged to use Environmental Protection Agency (EPA)-approved insect repellent with DEET, cover exposed skin, remain in screened-in areas, and treat clothing with permethrin. It is important to note that, when used as directed, EPA-registered repellants are safe for pregnant and breast-feeding women $[2 \bullet]$.

\section{Sexual Transmission}

The first documented case of sexual transmission occurred in 2008 when a scientist who contracted the infection while working in Senegal returned home and transmitted the virus to his wife, who had not left the USA in the prior year [6]. During the current outbreak, the CDC has confirmed six cases of sexual transmission in the USA, all of which were to the sexual partners of men returning from areas of active Zika transmission [7]. All reported cases have occurred following sexual contact with an infected man before the onset of or soon after the cessation of his symptoms. It remains unknown whether the partners of asymptomatic infected men can contract the virus. Additionally, the Zika virus has been isolated in the semen specimens from infected men for up to 62 days following the onset of symptoms; long after the virus was no longer detectable in the blood. The maximum duration of virus persistence in the semen is uncertain [8]. There have been no reports of sexual transmission from an infected woman to her partner.

\section{Prevention of Sexual Transmission}

The possibility of sexual transmission is of greatest concern in the pregnant partners of exposed men. Since the interval of potential transmission is uncertain, current statements from the Society for Maternal Fetal Medicine (SMFM) and the American Congress of Obstetricians and Gynecologists (ACOG) recommend the consistent use of barrier methods or abstinence from sexual intercourse for the duration of pregnancy for couples in which a man has traveled to or resides in an area with active Zika virus transmission [5••].

For couples attempting conception, it is recommended that barrier contraception is used or abstinence is observed for 6 months if a man had confirmed Zika infection or clinical illness or 8 weeks if a man traveled to an area with active viral transmission but is asymptomatic [9].

\section{Blood Transfusion}

During the Zika virus outbreak in French Polynesia, approximately $3 \%$ of donated blood samples tested positive for Zika virus [10]. To date there have been no cases of transmission via blood transfusion in the USA [2•]. There have been suspected cases in Brazil, but these have not been confirmed and are currently under investigation.

\section{Prevention of Transmission via Transfusion}

At this time, the incidence of Zika infection in the USA remains low suggesting a correspondingly low risk to the US blood supply. Regardless, the US Food and Drug Administration (FDA) still recommends that blood collection centers update their donor education materials to include instructing a donor to inform the center if they are diagnosed with or develop symptoms of Zika infection within 2 weeks following donation. They also recommend that donors defer for 4 weeks if they have a history of Zika virus infection, have traveled to an area with active Zika virus transmission, and have symptoms suggestive of Zika within 2 weeks of travel, have had sexual contact with a man diagnosed with Zika virus, have had sexual contact with a man who traveled to or resides in an area with active Zika transmission in the 3 months prior to the sexual contact, or are living in or traveling to an area with active Zika transmission [11]. Additionally, the FDA now recommends that blood collections cease in areas of the USA affected by active vector-borne transmission of Zika virus until screening of blood donation or pathogen reduction technology of blood components can be implemented [12]. 


\section{Vertical Transmission}

Zika virus has also been documented in amniotic fluid, fetal tissue from early missed abortions, placenta, and even the brains of newborns with microcephaly [13]. In April 2016, the Centers for Disease Control and Prevention (CDC) announced that there is now enough evidence to conclude Zika virus infection in pregnancy is a cause of microcephaly [2•]. The Zika virus has been known to be neurotropic since animal experiments dating back to the 1950s and has recently been shown to cause cell death and abnormal cell growth in human neuronal cell lines [14•]. In a study published in March 2016, Tang et al. infected multiple human cell lines with Zika virus and found more than $90 \%$ of cortical neural progenitor cells were damaged compared to much lower rates of injury in nonneuronal cells [14•], indicating that this important cell population in the developing embryonic brain is a direct target of Zika virus.

Further evidence strengthening the link between Zika virus infection in pregnancy and vertical transmission resulting in microcephaly comes from Brasil et al. in Rio de Janeiro who performed serum and urine testing in pregnant women at any gestational age who presented with a rash [15]. Real-time reverse transcription-polymerase chain reaction (RT-PCR) for the Zika virus was performed on specimens, and ultrasound was performed to evaluate for anatomic anomalies. Abnormal ultrasounds were seen in $29 \%$ of PCR-proven Zika infections. Sonographic findings included intrauterine growth restriction, microcephaly, cerebral calcifications, and decreased or absent amniotic fluid. Women infected at earlier gestational ages exhibited the most severe anomalies, but central nervous system malformations were also seen in infections diagnosed as late as 27 weeks.

Additionally, during the outbreak in French Polynesia, there were two reported cases of peripartum transmission. In these infants, Zika virus was detectable as early as 3 and 4 days post delivery while the serum sample collected the day of delivery was negative. This suggests perinatal rather than transplacental transmission. In both infants, the resulting illness was mild [16].

\section{Prevention of Vertical Transmission}

Unfortunately, there is currently no vaccine to prevent or treatment to cure Zika infection. Prevention strategies focus on avoidance of exposure, particularly during pregnancy. Currently, there is no evidence of a risk of infection to a fetus conceived after clearance of the virus from the mother's bloodstream. However, infection during early pregnancy is likely to result in the most severe pregnancy complications so a delay in conception is recommended. Thus, it is recommended that women who are diagnosed with Zika virus infection should delay conception for at least 8 weeks following symptom onset and men with Zika virus infection should wait 6 months after onset of symptoms before attempting conception $[5 \bullet \bullet, 9]$.

Other flaviviruses, including dengue virus, have been associated with vertical transmission through breastfeeding [17]. Zika virus has been isolated from the breast milk of infected mothers [18], implicating breastfeeding as a potential mechanism of transmission of the Zika virus. Nonetheless, transmission through breast milk has not been documented to date and any effects of neonatal infection are expected to be mild. In third world countries, the benefits of breastfeeding likely outweigh the potential risks and it is currently recommended that women with Zika continue to breastfeed [5••].

\section{Management of Pregnant Women}

Interpretation of laboratory testing available for the diagnosis or confirmation of Zika virus is complex. Testing is currently available at the $\mathrm{CDC}$ as well as several state health departments in the USA. During the first week following the onset of symptomatic illness, Zika virus can be diagnosed by performing RT-PCR on serum. Zika virus RNA can also be detected in urine for at least 2 weeks after the onset of symptoms and the CDC now recommends that Zika virus RT-PCR be performed on urine collected $<14$ days after onset of symptoms in patients with suspected Zika virus disease [19]. Urine testing should be performed in conjunction with serum testing if specimens collected less than 7 days after symptom onset are used [19]. Virus specific IgM antibodies may be detectable greater than 4 days after the onset of illness and persist for approximately 2 to 12 weeks. IgM antibodies against Zika virus, however, have strong cross reactivity with other flaviviruses and may generate false positive results [9]. Zika virus is a nationally notifiable condition and all health care providers are encouraged to report suspected Zika cases to their local health department. Guidelines for the collection and submission of fetal tissues and body fluids for Zika virus testing can be found on the CDC website (http://www.cdc. gov/zika/hc-providers/diagnostic.html).

\section{Initial Evaluation in Areas Without Active Mosquito-Borne Transmission}

All healthcare providers should evaluate their pregnant patients for potential exposure at each visit. This should include assessment of the travel history of the patients themselves as well as their sexual partners and elicitation of symptoms consistent with Zika infection.

Pregnant women with symptoms suggestive of Zika during or within 2 weeks of travel should have Zika testing with RTPCR and IgM serologies with further management dictated by these results. Asymptomatic women with a history of travel 
should be tested with Zika virus antibody IgM 2 to 12 weeks after exposure. In this scenario, a negative result cannot definitively rule out Zika infection but would indicate that a recent Zika virus infection did not occur and may obviate the need for serial ultrasonography. Fetal ultrasonography focusing on intracranial calcifications or microcephaly should also be obtained 3 to 4 weeks after exposure or symptom development $[5 \cdot \bullet, 9]$. The most recent algorithm for testing a pregnant woman with possible Zika virus exposure who is not residing in an area with active Zika virus transmission can be found at http://www.cdc.gov/mmwr/volumes/65/wr/mm6512e2. htm\#F1_down [9].

\section{Initial Evaluation in Areas With Active Mosquito-Borne Transmission}

Women residing in areas with ongoing Zika transmission should be monitored for symptoms at each visit. Should clinical illness develop, serum RT-PCR and IgM should be obtained. If the woman remains asymptomatic, current recommendations include obtaining IgM at her first prenatal visit with repeated serologic testing in the late second trimester. Asymptomatic women should also receive an ultrasound at 18-20 weeks to evaluate for the presence of anomalies [5••, 9].

\section{Further Testing}

In the case of positive maternal serology and/or PCR, serial ultrasounds are recommended every 3-4 weeks. The natural history of in utero Zika virus infection and the time from exposure to clinical manifestations remains unknown. For this reason, additional ultrasound examinations should be considered even in the case of asymptomatic exposure, although the time interval at which it should occur is uncertain $[5 \bullet \bullet, 9]$. If anatomic anomalies like microcephaly or intracranial calcifications are discovered on ultrasound, amniocentesis for Zika virus testing may be considered.

\section{Neonatal Evaluation and Outcomes}

Microcephaly refers to the presence of an abnormally small head for gestational age and is usually indicative of an underlying problem with brain development. It can exist alone or as a component of a congenital syndrome, and can be diagnosed in utero or postnatally. Affected children may experience seizures, developmental delay, intellectual disabilities, hearing loss, and vision problems. The etiology of microcephaly is complex and multifactorial, encompassing environmental exposures, infectious agents, potentially including Zika virus, and genetic causes. Currently, much of what is known about the neonatal effects of congenital Zika virus infection is derived from case reports and past experience with maternal rubella and cytomegalovirus infection.

Patient counseling on ultrasonographic findings is complicated by the fact that no standardized or consistent case definitions exist for the monitoring of microcephaly in utero. In a statement from SMFM, the difficulties in differentiating between a constitutionally small head and pathologic microcephaly were addressed [20]. SMFM recommends defining isolated fetal microcephaly as a head circumference of 3 standard deviations or more below the mean for gestational age. The statement further recommends that if the head circumference is greater than 2 standard deviations below the mean, a careful evaluation of fetal intracranial anatomy should be performed and, and if normal, the ultrasound should be repeated in 3-4 weeks [20]. Despite efforts at improving the antenatal diagnosis of microcephaly, the full spectrum of neonatal outcomes from in utero Zika infection has not yet been elucidated and there is no ultrasonographic marker that reliably predicts the subsequent severity of symptoms that will be experienced by the neonate. Further, there is currently no known treatment available to ameliorate the affects of or halt the progression of the virus in the fetus. Detailed counseling on the spectrum of pregnancy outcomes in Zika infection ranging from early pregnancy loss or fetal death to birth of a neonate with microcephaly and possible subsequent learning disabilities, hearing loss, and ocular abnormalities is required. Referral to a maternal fetal medicine specialist is recommended for any woman suspected to have Zika virus in pregnancy.

In contrast to fetal infection, it is likely that de novo infections of infants would result in a minor clinical illness similar to that seen in non-pregnant adults [21].

\section{Conclusions}

While the data available on Zika virus and its potential effects on pregnancy is rapidly increasing and evolving, there are still many uncertainties plaguing the medical profession in general and obstetrical providers specifically. Professional societies including SMFM, ACOG, and CDC have published practice guidelines indicating which populations of pregnant women should be tested for Zika virus and the frequency of ultrasonographic assessments with which these at risk pregnancies should be followed. Nonetheless, many of the queries most often posed by concerned women remain unanswered. For instance, the rate of vertical transmission from mother to fetus, the risk of microcephaly and other central nervous system anomalies in fetuses when maternal infection is proven, and the time from exposure or infection to clinical manifestations including ultrasound anomalies are unknown. Further studies are needed to answer these important questions and direct the counseling of pregnant women who may be affected. 


\section{Compliance with Ethical Guidelines}

Conflict of Interest D. G. Fiorentino and F. J. Montero declare that they have no conflict of interest.

Human and Animal Rights and Informed Consent This article does not contain any studies with human or animal subjects performed by any of the authors.

\section{References}

Papers of particular interest, published recently, have been highlighted as:

- Of importance

•- Of major importance

1. Oster AM, Brooks JT, Stryker JE, et al. Interim guidelines for prevention of sexual transmission of Zika virus-United States, 2016. MMWR Morb Mortal Wkly Rep. 2016;65(5): 120-1. doi:10.15585/mmwr.mm6505e1.

2. Centers for Disease Control and Prevention (CDC). About Zika virus disease. 2016. http://www.cdc.gov/zika/about/ This resource is the current official guidelines for the prevention of transmission of Zika and the most up to date information about its spread.

3. Gulland A. Zika virus is a global public health emergency, declares WHO. BMJ. 2016;352:i657. doi:10.1136/bmj.i657.

4. Petersen LR, Jamieson DJ, Powers A, Honein M. Zika virus. New Engl J Med. 2016;374(16):1552-63. doi:10.1056/NEJMra1602113.

5.• ACOG and SMFM Practice Advisory. Updated Interim Guidance for Care of Women of Reproductive Age During a Zika Virus Outbreak. 2016. http://www.acog.org/About-ACOG/NewsRoom/Practice-Advisories/Practice-Advisory-Interim-Guidancefor-Care-of-Obstetric-Patients-During-a-Zika-Virus-Outbreak. This article provides the most recent recommendations for the screening and management of pregnant women with Zika exposure and dictates how obstetricians in the USA are responding to the outbreak

6. Foy BD, Kobylinski KC, Foy JLC, et al. Probable non-vector-borne transmission of Zika virus, Colorado, USA. Emerg Infect Dis. 2011;5:880-2. doi:10.3201/eid1705.101939.

7. Oster AM, Russel K, Stryker JE, et al. Update: Interim guidance for prevention of sexual transmission of Zika virus - United States, 2016. MMWR Morb Mortal Wkly Rpt. 2016;65(12):323-5. doi:10.15585/mmwr.mm6512e3.

8. Atkinson B, Hearn P, Afrough B, et al. Detection of Zika virus in semen [letter]. Emerg Infect Dis. 2016;22(5):940. doi:10.3201/eid2205.160107.

9. Petersen EE, Polen KN, Meaney-Delman D, et al. Update: interim guidance for health care providers caring for women of reproductive age with possible Zika virus exposure-United States, 2016. MMWR Morb Mortal Wkly Rep. 2016;65(12):31522. doi:10.15585/mmwr.mm6512e2.

10. Musso D, Nhan T, Robin E, Roche C, Bierlaire D, Zisou K, et al. Potential for Zika virus transmission through blood transfusion demonstrated during an outbreak in French Polynesia, November 2013 to February 2014. Euro Surveill. 2014;19(14).

11. Food and Drug Administration. Recommendations for donor screening, deferral, and product management to reduce the risk of transfusion-transmission of Zika Virus- Guidance for industry. 2016. http://www.fda.gov/BiologicsBloodVaccines/Guidance ComplianceRegulatoryInformation/Guidances/default.htm.

12. Vasquez AM, Sapiano MR, Basavaraju SV, Kuehnert MJ, RiveraGarcia B. Survey of blood collection centers and implementation of guidance for prevention of transfusion-transmitted Zika virus infection-Puerto Rico, 2016. MMWR Morb Mortal Wkly Rep. 2016;65:375-8. doi:10.15585/mmwr.mm6514e1.

13. Martines RB, Bhatnagar J, Keating MK, et al. Notes from the field: evidence of Zika virus infection in brain and placental tissues from two congenitally infected newborns and two fetal losses-Brazil, 2015. MMWR Morb Mortal Wkly Rep. 2016;65(6):159-60. doi:10.15585/mmwr.mm6506e1.

14. Tang H, Hammack C, Ogden SC, et al. Zika virus infects human cortical neural progenitors and attenuates their growth. Cell Stem Cell. 2016. doi:10.1016/j.stem.2016.02.016. This article provides scientific evidence supporting the association of Zika virus infection in utero and congenital brain anomalies.

15. Brasil P, Pereira J, Jose P, Raja Gabaglia C, Damasceno L, Wakimoto $\mathrm{M}$, et al. Zika virus infection in pregnant women in Rio de Janeiro-preliminary report. N Engl J Med. 2016. doi:10.1056/NEJMoa1602412.

16. Besnard M, Lastère S, Teissier A, Cao-Lormeau VM, Musso D. Evidence of perinatal transmission of Zika virus, French Polynesia, December 2013 and February 2014. Euro Surveill. 2014;19(13).

17. Barthel A, Gourinat AC, Cazorla C, Joubert C, Dupont-Rouzeyrol M, Descloux E. Breast milk as a possible route of vertical transmission of Dengue virus? Clin Infect Dis. 2013;57(3):415-7. doi:10.1093/cid/cit227.

18. Dupont-Rouzeyrol $\mathrm{M}$ et al. Infectious Zika viral particles in breastmilk. The Lancet. 2016;387(10023):1051. doi:10.1016/S0140-6736(16)00624-3.

19. Interim guidance for Zika virus testing of urine-United States, 2016. MMWR Morb Mortal Wkly Rep. 2016;65. doi:10.15585/mmwr.mm6518e.

20. Society for Maternal Fetal Medicine Statement: Ultrasound screening for fetal microcephaly following Zika exposure. AJOG February 2016. http://www.ajog.org/pb/assets/raw/Health\%20 Advance/journals/ymob/SMFM\%20Statement_Feta1\%20 microcephaly.pdf.

21. Centers for Disease Control and Prevention. Zika Virus: For Health Care Providers: Clinical Evaluation \& Disease. http://www.cdc. gov/zika/hc-providers/clinicalevaluation.html. 